\title{
Selectorate Theory, the Democratic Peace, and Public Goods Provision
}

Article · May 2012

READS

238

7 authors, including:

Nicholas Nicoletti

Missouri Southern State University

2 PUBLICATIONS 0 CITATIONS

SEE PROFILE
Keith Krehbiel

Stanford University

87 PUBLICATIONS 3,049 CITATIONS

SEE PROFILE 


\title{
Selectorate Theory, the Democratic Peace, and Public Goods Provision*
}

\author{
Philip Arena ${ }^{\dagger}$ \\ and \\ Nicholas P. Nicoletti ${ }^{\ddagger}$
}

April 9, 2012

\begin{abstract}
We show that without a few peculiar modeling choices that are not justified by the core assumptions of the theory, selectorate theory neither unambiguously predicts the democratic peace nor that leaders of more inclusive regimes will rely upon the provision of public goods to remain in office. We illustrate these claims using relatively simple models that incorporate the core assumptions of their theory while avoiding modeling choices we believe to be less appropriate. We also discuss reasons to be skeptical that the historical record has in fact indicated that there is any relationship between democracy and public goods provision, focusing on recent evidence with respect to poverty and environmental degradation. We argue for a revised version of selectorate theory, one that continues to emphasize the importance of the size of the winning coalition, yet we believe provides a more realistic picture of democratic politics.
\end{abstract}

${ }^{*}$ We would like to thank Erik Gartzke, Errol Henderson, Brenton Kenkel, Keith Krehbiel, Nolan McCarty, and the participants of the 2010 Annual Meeting of the Peace Science Society (International) for helpful comments and suggestions. Any remaining errors are our own.

${ }^{\dagger}$ Assistant Professor, Department of Political Science, University at Buffalo, SUNY. parena@buffalo.edu

${ }^{\ddagger}$ Ph.D. Student, Department of Political Science, University at Buffalo, SUNY. npn@buffalo.edu 
The claim that pairs of democracies are less likely to come into armed conflict with one another than the average pair of states is widely accepted in international relations. While there may be other explanations for the absence of conflict amongst Western states, such as the adoption of liberal economic policies and membership in international governmental organizations, most scholars believe that democracy itself is an important force for peace. ${ }^{12}$ One reason that scholars remain skeptical of attempts to dispute the empirical association between democracy and peace is that the theoretical explanations for why democracies ought to be less conflict prone have proven quite fruitful, with many of their additional observable implications having been supported. For many, the democratic peace is no longer an isolated, if important, empirical finding, but the foundation of a mature research program. ${ }^{3}$

In many ways, selectorate theory lies at the heart of this research program. It not only accounts for many patterns with respect to international conflict (Bueno de Mesquita et al. 1999, Morrow et al. 2006), but also international cooperation (Bueno de Mesquita and Smith 2007, 2009) ${ }^{4}$ the provision of public goods, institutional change, leadership tenure, and many other domestic level outcomes (Bueno de Mesquita et al. 2003). Until recently, it was one of few explanations for the democratic peace that had been formalized. ${ }^{56}$

\footnotetext{
${ }^{1}$ On the impact of economic policies and IGOs, see, inter alia, Anderson and Souva (2010), Dorussen and Ward (2008, 2010), Gartzke, Li and Boehmer (2001) and, especially, Gartzke (2007). Oneal and Russett (1997), Oneal, Russett and Berbaum (2003), Russett and Oneal (2001) view these factors as complementary.

${ }^{2}$ Note that Henderson (2009) provides evidence, using a research design similar to that of Russett and Oneal (2001), that the democratic peace does not hold in any region outside the West. Separate analysis (not reported) reveals that the evidentiary basis is even more narrow than that, disappearing once one omits the US, UK, France, Germany, Italy and Austria. Thus, we may be observing little more than the transformation of relations between the European great powers following WWII. See also Gibler (2007), who presents evidence that democracy is a consequence of the resolution of territorial disputes, and thus peace may cause democracy rather than vice versa.

${ }^{3}$ See Ray $(1995,2003)$ for such claims.

${ }^{4}$ McGillivray and Smith (2008) builds upon selectorate theory to explain still further important patterns of international cooperation. However, their argument is nonetheless distinct from selectorate theory.

${ }^{5}$ Some authors have suggested that audience costs might account for the democratic peace (Fearon 1994,

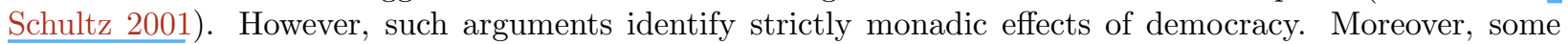
studies suggest that the relationship between regime type and the ability to generate audience costs is not linear (Slantchev 2006, Weeks 2008). Finally, since all regimes can employ military mobilization, it is not obvious why the ability to generate audience costs should afford democracies any particular advantage even if they were better able to generate them (Slantchev 2005).

${ }^{6}$ However, see Debs and Goemans (2010), Fearon (N.d.), who provide alternative formal explanations of the democratic peace that yield additional observable implications. See also Patty and Weber (2006) and Jackson and Morelli (2007), both of whom argue that democracies are less prone to bad decision making.
} 
Recently, Clarke and Stone (2008) argued that the proxy variables for $W$ and $S$ constructed by Bueno de Mesquita et al. (2003) largely do not account for the relationship between democracy and the outcome variables the authors analyze. Yet, even if we accept their claim, ${ }^{7}$ one might wonder whether this reflects an actual shortcoming of selectorate theory rather than the crude nature of the measurement of the theory's key variables.

We seek to demonstrate that the problem is in fact fundamental to the theory. Specifically, a few particular modeling choices in Bueno de Mesquita et al. (2003, 1999) prove critical for their primary conclusions with respect to democratic governance. These choices are neither required, nor justified, by the core assumptions of selectorate theory. They are also, we believe, difficult to defend substantively. That they appear necessary for some of the most celebrated results is therefore troubling. We also discuss reasons why we should be skeptical of the apparent relationship between democracy and public goods provision, which we believe the historical record does not unambiguously suggest democracies are more inclined to do than non-democracies. At least not when public goods are defined properly.

To develop our theoretical claims, we analyze two simple formal models that are designed to capture what the authors indicate are the critical assumptions of selectorate theory while avoiding some of what we believe to be more problematic features of their models. ${ }^{8}$ The two features that we focus upon are: 1) though the model in Bueno de Mesquita et al. (1999) includes an outcome referred to as negotiations, the players do not negotiate over the issues in dispute in the sense most scholars use the term, as the terms are not chosen by the actors themselves, nor do negotiated agreements require consent from both parties, and 2) with respect to the selection of a leader, Bueno de Mesquita et al. (2003) assume that the outcome preferred by the selectorate collectively obtains, without specifying any connection between the choices made by individual members of the selectorate and the aggregate outcome.

\footnotetext{
${ }^{7}$ See the response (Morrow et al. 2008).

${ }^{8}$ When discussing the provision of public goods, we primarily focus on the model outlined in the first three chapters of Bueno de Mesquita et al. (2003). When discussing the democratic peace, we focus primarily on Bueno de Mesquita et al. (1999). However, our critique of the decision rule for selecting leaders applies also to Bueno de Mesquita et al. (1999), and negotiations are modeled similarly in both Bueno de Mesquita et al. (1999) and chapter 6 of Bueno de Mesquita et al. (2003).
} 
We begin by demonstrating that even if we assume that domestic politics operates as described by Bueno de Mesquita et al. (1999), it is still not clear that we should expect pairs of democracies to be less likely to come into conflict than other pairs of states. The model in Bueno de Mesquita et al. (1999) does not allow either side to have any control over the terms of a negotiated agreement. Further, it is not necessary for both sides to accept an agreement for it to enter into force. In essence, the model in Bueno de Mesquita et al. (1999) treats negotiations as randomly determined outcomes that one side can unilaterally impose upon the other against their will, despite a longstanding belief that any state may choose to use force at any time if it so pleases (Waltz 1979).

Using a simpler model than the one analyzed by Bueno de Mesquita et al. (1999), one in which we take for granted the claim that political survival requires some leaders to try harder in war than others, we show that we can derive the democratic peace when we model negotiations the way they did. Next, we show that no relationship between domestic politics and war follows from such a model once we allow for negotiations using the more flexible ultimatum bargaining protocol popularized by Fearon (1995), since war never occurs in equilibrium under complete information.

If we assume incomplete information, which Bueno de Mesquita et al. (1999) did not, war naturally is possible. But our results still do not appear consistent with the democratic peace. Rather, they strangely indicate that peace is either most likely or least likely in dyads containing one autocracy and one democracy, relative to all other combinations. Whether such dyads are especially peaceful or prone to conflict depends upon which is the initiator. This result stands in stark contrast to extant empirical findings (Bennett 2006).

We next demonstrate the core assumptions of selectorate theory do not unambiguously indicate that democracies will choose to provide public goods while in autocracies "good policy is bad politics", as Bueno de Mesquita et al. (2003) frequently claim. Bueno de Mesquita et al. (2003) assume that the only relevant calculation is whether members of the incumbent leader's current winning coalition would receive more goods if they retain the 
leader or if they replace the leader with some challenger. This, in effect, forces members of the selectorate to ignore the very defining feature of public goods - that they do not individually need to support the incumbent in order to enjoy any public goods she will provide if retained, though they must do so in order to receive private goods. As we demonstrate below, if individual members of the selectorate calculate the difference in the amount of goods they personally expect to receive as a result of their individual choice of whom to support and nothing else, then the leader may provide precisely zero goods in equilibrium.

Naturally, we might expect that other considerations enter into the decision of whom to support, at least at the margins. We thus allow members of the selectorate to receive arbitrarily small non-material rewards or punishments for expressing support for the incumbent leader. ${ }^{9}$ These non-material rewards may reflect ideological agreement, ethnic kinship, or other factors. After we introduce these terms, in the form of private information held by each member of the selectorate, we unsurprisingly find that leaders are required to distribute resources in order to retain office. Leaders can no longer rely on the fact that there is no $m a-$ terial incentive to vote against a candidate who is certain to win even when that candidate does not promise to deliver any resources to anyone.

However, the conditions under which leaders choose to allocate resources in the form of public goods are very restrictive. So long as individual members of the selectorate cannot expect to reduce the probability that the leader retains office by a substantial amount as a result of their individual choice to support the challenger, the leader has no incentive to provide public goods, which everyone knows they will receive should the leader win regardless of whether they individually supporter her. Since it is exceedingly implausible for any one voter to have a non-trivial impact on the outcome of an election in a modern electoral democracy, this version of our model, which we believe reflects the core assumptions of selectorate theory better than the next two models we consider, cannot explain why we would observe the provision of public goods in such regimes.

\footnotetext{
${ }^{9}$ This is not dissimilar to the affinity term that Bueno de Mesquita et al. (2003) use to identify which members of the selectorate the leader will include in her winning coalition.
} 
Nonetheless, while this represents a more significant departure from the core assumptions of selectorate theory, we also consider an extension of the model where the expressive utility members of the selectorate receive is explicitly a function of whether the incumbent provides public goods, rather than representing non-material reasons for the selector to feel affinity for the leader. Here, we unsurprisingly find that leaders have an incentive to produce public goods. All leaders. Thus, this version of the model explains why democracies might provide public goods, but it does so by raising the puzzle of why non-democracies do not.

To address this, we also consider one last version of the model in which we assume that within all societies, there is a subset of the selectorate that derives expressive utility from supporting leaders on the basis of non-material factors, and another subset whose expressive utility depends upon the leader's provision of public goods. We show that if the latter, more public-spirited set of selectors, is arbitrarily large but not completely dominant, it is impossible for leaders of democracies to construct a winning coalition without providing public goods while it is possible for some autocrats to do so. However, we stress that this is at best a poor substitute for the arguments of Bueno de Mesquita et al. (2003), who, to their credit, only argue that the low rates of public goods provision in non-democratic systems reflects simple political necessity. ${ }^{10}$

We believe that neither of these two versions of the model provides a great fit to the empirical record. Instead, we argue that focusing on the size of the winning coalition can indeed be very useful for understanding the behavior of leaders. But we believe that there is an important distinction between the claim that all leaders seek to retain office by providing private goods to their winning coalition, which happens to mean a good proportion of the populace in some cases, and the claim that leaders of regimes with small winning coalitions rely on private goods while leaders of regimes with large winning coalitions rely on public goods - goods which by their very definition benefit the entire populace.

\footnotetext{
${ }^{10}$ Their oft-repeated claim that "good policy is bad politics" illustrates that leaders of regimes with small winning coalitions cannot afford to provide public goods even if they desire to, since doing so would necessarily reduce the amount of private goods allocated to their key supporters, who would those overthrow the leader.
} 
For this reason, we stress that the theoretical argument presented by Bueno de Mesquita et al. (2003) depends critically upon the fact that public goods are non-rival and nonexcludable. ${ }^{11}$ Yet very few of the outcome variables they consider, such as the number of hospital beds, rates of female secondary education, immunizations, etc, can plausibly be argued to be either non-rival or non-excludable. This is an important distinction, because such measures also do not tell us about whether democracies primarily only provide benefits to politically important members of society, of which there are by definition a great many more than in most non-democratic regimes, or whether they are in fact more likely to produce goods that benefit even the worst off in society.

To illustrate the importance of this distinction, we briefly discuss recent research on the the relationship between regime type and true public goods provision. Specifically, we focus on a prominent recent study that indicates that democracy is not in fact good for the poor (Ross 2006) and recent evidence that democracies are not better stewards of the environment. These studies also provide very good reason to believe that empirical tests that purport to show that democracy benefits even the least of its citizens suffer from severe bias due to the non-random pattern of missing data. While we acknowledge that this remains an active area of research, we believe there is strong evidence so far that democracy empowers a large proportion of its people, which is certainly preferable to empowering a rare few, but does not necessarily work for everyone. We argue that even in democracies, the political process is fundamentally a process of creating winners and losers. ${ }^{12}$

We begin with a brief review of selectorate theory. We then present a simple model of crisis bargaining, followed by a simple model of leader selection. We then discuss the implications of our analysis before concluding.

\footnotetext{
${ }^{11}$ They never claim that even in systems with large winning coalitions leaders wish to make everyone happy, only that it is more economically efficient to do so by producing goods that benefit everyone in their attempt to reward members of their winning coalition.

${ }^{12}$ Interestingly, some of the same authors have elsewhere offered a novel solution to the paradox of voting that is in many respects consistent with the arguments we develop here. Specifically, Smith and Bueno de Mesquita (N.d.) argue that even if an individual's vote is not likely to be pivotal with respect to which candidate assumes office, it may be pivotal in determining whether their precinct receives patronage. Thus, voters are motivated by the impact of their individual decision on access to particularistic goods.
} 


\section{Selectorate theory and the Democratic Peace}

The literature on the democratic peace grew out of the empirical association between joint democracy and a reduced likelihood of conflict. ${ }^{13}$ Scholars did not propose well-developed theoretical explanations of the pattern until later. Two general approaches have been offered to account for the finding: norms and institutions. Recently many scholars have gravitated towards the institutional approach, for various reasons. ${ }^{14}$ One prominent variant of the institutional approach emphasizes accountability. According to this view, all leaders are assumed to be self-interested and to value retaining office first and foremost. Democrats are expected to behave differently than autocrats, irrespective of their commitment to normative values, simply because they rely upon a broader base of support to remain in office than do autocrats, and thus must satisfy larger segments of the populace in order to advance their own personal goal of retaining power. This is assumed to require promoting the public good.

To date, the most prominent theoretical explanation of the democratic peace that emphasizes the role of domestic institutions is selectorate theory (Bueno de Mesquita et al. 2003, 1999). Selectorate theory begins from the simple and appealing claim that much of the behavior of states can be characterized by the relative proportion of a society's population that falls into each of two groups: the selectorate, $S$, and the winning coalition, $W$. The selectorate is the subset of the population who directly influence the selection of a leader. In an electoral democracy, the selectorate is simply the electorate, while in military juntas it largely consists of senior officers. The winning coalition is the minimal subset of $S$ whose support is sufficient to allow one to take office. ${ }^{15}$ In electoral democracies, $W$ typically constitutes a large proportion of $S .{ }^{16}$ In other regimes, $W$ may be countably small.

\footnotetext{
${ }^{13}$ Babst (1964), Bremer (1992), Maoz and Russett (1993).

${ }^{14}$ See Henderson (2002), Rosato (2003) and Reiter and Stam (2002) for critiques of the normative approach. However, see also Huth and Allee (2002) and Danilovic and Clare (2007).

${ }^{15}$ Note that this need not be the same as the subset of $S$ who, in practice, actually do support a candidate, which Morrow et al. (2008) term the supporting coalition to distinguish it from the winning coalition. Thus selectorate theory suggests that even if a leader gathers the votes of $55 \%$ of the populace in an election, only some subset of those supporters properly belong to $W$.

${ }^{16}$ However, $W$ need not be half of $S$. In first-past-the-post systems, leaders require half of the votes in half of the districts. In proportional representation systems, minority governments are often formed.
} 
The authors argue that the primary implication of selectorate theory is that leaders who must satisfy larger winning coalitions are more likely to rely on the distribution of public goods to satisfy their supporters rather than private goods, while the opposite is true of leaders of systems where $W$ is small. A secondary implication is that as the ratio of $W$ to $S$ increases, the leader retains fewer resources for herself. As democratic states typically have both a large $W$ and a large ratio of $W$ to $S$, they are expected to produce larger quantities of public goods, and their leaders are more likely to be removed from office following policy failures that constitute exogenous shocks to the leader's resource base. Extensive empirical analyses seemingly indicate that democracy is associated with many positive outcomes, and that leaders of democracies are more susceptible to removal from office following policy failure (Bueno de Mesquita et al. 2003). ${ }^{17}$

Selectorate theory has been applied to a wide range of outcomes beyond the democratic peace, the provision of public goods, and the average duration of a leader's tenure. The authors have applied selectorate theory to explain tax rates, corruption, and other domestic level factors (Bueno de Mesquita et al. 2003). With respect to international relations, the authors offer novel claims about which states are more likely to pursue regime change as a war aim (Bueno de Mesquita et al. 2003, Morrow et al. 2006), and which states are most likely to provide foreign aid, how much they choose to give, and to whom (Bueno de Mesquita and Smith 2007, 2009). McGillivray and Smith (2006, 2008) draw on selectorate theory in developing their theory of Leader-Specific Punishments, which explains patterns in sovereign debt, trade, economic sanctions, and other outcomes.

Put simply, few other theories have proven to be as rich, parsimonious, and influential. It is therefore important to highlight what we believe to be significant shortcomings.

\footnotetext{
${ }^{17}$ The authors argue that $W$ and $S$ capture independent information than measures of democracy versus autocracy, despite earlier claiming that their theory explains the empirical relationship between democracy and peace (Bueno de Mesquita et al. 1999). See Clarke and Stone (2008) for a critique of the empirical evidence that $W$ and $S$ explain outcomes better than standard measures of democracy. However, given that the empirical proxies for $W$ and $S$ used by the authors are as crude as they are, it is difficult to know what we can infer from the performance of these proxies relative to measures such as Polity. For example, simple cross-tabs reveal that $W$ is larger than $S$, despite being a subset thereof, in nearly $10 \%$ of observations.
} 


\section{Negotiation and the Democratic Peace}

In this section, we demonstrate that the non-standard bargaining protocol in the model analyzed by Bueno de Mesquita et al. (1999) is critical to producing their results. Naturally, this would not itself be much of a concern if the authors provided strong theoretical justification for making the assumptions they have about the bargaining protocol. But they do not, and the bargaining protocol in their model is difficult to justify substantively. Specifically, while the authors treat the issue in dispute as divisible, the terms of a negotiated agreement are not chosen by the players but are instead treated as exogenous. Moreover, negotiated agreements can be unilaterally imposed by one side regardless of whether the other would prefer war to abiding by such terms. Though treating the good in dispute as divisible addresses one part of the argument presented in Fearon (1995), their approach does not address the more fundamental point Fearon raised: the costs of war are sufficient to ensure the existence of a range of agreements that both sides prefer to war. We analyze a simpler model, one that assumes one of the key results that they derive from their model, thereby stacking the deck in favor of reproducing their argument. We show that the democratic peace is implied by our model under their bargaining protocol, but is not apparently compatible with the ultimatum bargaining protocol, which has become one of the standard protocols in the literature.

Specifically, we do not explicitly model reselection following the international crisis. Nor do we explicitly model the selection of war effort, an important component of Bueno de Mesquita et al. (1999). However, we take as given that patterns mirroring those derived by Bueno de Mesquita et al. (1999) obtain with respect to the relationship between domestic institutions and the optimal war effort chosen by each side. This allows us to focus attention strictly upon the importance of the author's choice of bargaining protocol.

More formally, the leader of state 1 , denoted $L_{1}$, and the leader of state $2, L_{2}$, contest the division of some good, whose value is normalized to 1 . We assume that the good in dispute is of value because it can be used to increase each leader's resource base. ${ }^{18}$

\footnotetext{
${ }^{18}$ Note Bueno de Mesquita et al. (1999) argue that control of the good in dispute is a public good. However,
} 
We first assume the game begins with $L_{1}$ choosing between war and a negotiated agreement. We do not allow $L_{2}$ the opportunity to reject the agreement. Under a negotiated agreement, $L_{1}$ receives $x \in[0,1]$ while $L_{2}$ receives $1-x$, where the value of $x$ is exogenously determined. This mirrors the bargaining protocol in Bueno de Mesquita et al. (1999).

We will then consider a version of the model with a more flexible bargaining protocol. In this version, $L_{1}$ is allowed to choose the value of $x$ and $L_{2}$ is allowed to decide whether to accept or reject $L_{1}$ 's proposal. The payoffs for a negotiated settlement in this version are the same: $L_{1}$ receives $x$ and $L_{2}$ receives $1-x$. The difference is simply that in this version, the size of $x$ is chosen by $L_{1}$ and enacted if and only if $L_{2}$ is willing to accept such a division of the good, whereas Bueno de Mesquita et al. (1999) allow $L_{1}$ to impose $x$ on $L_{2}$.

Wars are modeled identically in both versions of the model. $L_{1}$ wins the war with probability $p$ and $L_{2}$ wins with probability $1-p$. Winning is assumed to be worth $1-c_{i}$ and losing worth $-c_{i}$, where $c_{i} \in[0,1]$ reflects $i$ 's subjective loss of utility for incurring the costs of war. Let $p=\frac{e_{1}}{e_{1}+e_{2}}$, where $e_{i}>0$ is the amount of resources leader $L_{i}$ devotes to the war effort. We assume that $L_{i}$ selects $e_{i}$ to ensure that $p$ maximizes their probability of survival in office, subject to budget constraints. We assume war outcomes influence survival. ${ }^{19}$

More formally, let the optimal war effort be denoted by $e_{i}^{*}$, and let $e_{i}^{*}$ increase as $W_{i}$ increases or $S_{i}$ decreases, and let the magnitude of this effect increase as $W_{j}$ increases or $S_{j}$ decreases $\forall i \neq j$. Thus $\frac{\partial e_{i}^{*}}{\partial W_{i}}>0, \frac{\partial e_{i}^{*}}{\partial S_{i}}<0, \frac{\partial^{2} e_{i}^{*}}{\partial W_{i} \partial W_{j}}>0$ and $\frac{\partial^{2} e_{i}^{*}}{\partial S_{i} \partial S_{j}}<0$.

Substantively, these assumptions indicate that leaders of more open regimes can be expected to try harder to win the wars they choose to fight, and leaders of all regimes are forced to try harder to ensure the same probability of victory when facing an opponent who also has domestic political incentives to try harder. These assumptions echo key results derived in Bueno de Mesquita et al. (1999).

we need only assume that control of the good enables the leader to shore up domestic support, which would be true if it expands the resource base irrespective of whether the leader will then use the additional resources to provide more public goods or more private goods.

${ }^{19}$ However, the relationship between leadership survival, war outcomes, and domestic institutions may differ substantially from that envisioned by selectorate theory. See, for example, Goemans (2000) and Chiozza and Goemans (2004). 
Let us further assume that the cost $L_{i}$ incurs under the optimal war effort is a strictly convex function of $L_{i}$ 's war effort. That is, we assume that the cost of war increases at an increasing rate, $\frac{\partial c_{i}}{\partial e_{i}}>0$ and $\frac{\partial^{2} c_{i}}{\partial^{2} e_{i}}>0$. This indicates that there are diminishing returns to scale, as we might expect given that $p$ increases at a decreasing rate as $e_{1}$ increases and decreases at a decreasing rate as $e_{2}$ increases. Since $c_{i}$ reflects the subjective loss of utility associated with war under a given level of effort, this should be uncontroversial.

The solution for each version of the model is straightforward. Note that since we do not explicitly model reselection or the choice of war effort, the version of the model with the bargaining protocol equivalent to that in Bueno de Mesquita et al. (1999) is not gametheoretic, but simply decision theoretic. $L_{1}$ chooses negotiation if and only if $x \geq p-c_{1} \equiv \hat{x}$.

The democratic peace follows readily from the assumptions we made above. That is, $\frac{\partial e_{1}^{*}}{\partial W_{1}}>0, \frac{\partial^{2} e_{1}^{*}}{\partial W_{1} \partial W_{2}}>0, \frac{\partial c_{1}}{\partial e_{1}}>0$ and $\frac{\partial^{2} c_{1}}{\partial^{2} e_{1}}>0$ ensure that $c_{1}$ increases, and therefore $\hat{x}$ decreases, as $W_{1}$ increases, and that effect itself increases as $W_{2}$ increases. Therefore, the conditions under which war occurs become more difficult to satisfy as the size of the minimum winning coalitions in both states increase. If a large $W$ is associated with democracy, then pairs of democracies will be less likely to come into conflict with one another than other pairs of states. Further, the impact of $L_{1}$ 's institutions depends upon $L_{2}$ 's, consistent with the longstanding claim that the democratic peace is inherently dyadic.

Now let us turn to the second version, where $L_{1}$ chooses the size of $x$ and $L_{2}$ decides whether to accept $L_{1}$ 's proposed division of the good or reject it and go to war. $L_{2}$ accepts any $x \leq p+c_{2}$. Since $U_{L_{1}}\left(x=p+c_{2}\right) \geq E U_{L_{1}}\left(x>p+c_{2}\right)$ is equivalent to $p+c_{2} \geq p-c_{1} \Leftrightarrow$ $c_{1}+c_{2} \geq 0, L_{1}$ must always prefer to set $x=p+c_{2}$, which $L_{2}$ is certain to accept. Since this model always predicts peace, it cannot predict any relationship between $W_{1}, W_{2}$ and peace.

This informal proof is, of course, identical to that in Fearon (1995), which illustrates our argument that Bueno de Mesquita et al. (1999) were able to claim that selectorate theory explains the democratic peace only because they sidestepped Fearon's argument about the inefficiency of war through employing their particular bargaining protocol. 
Naturally, even though Bueno de Mesquita et al. (1999) assumed complete information, one might reasonably wonder what the equilibria to this model look like if we assume incomplete information, the primary explanation for war offered by Fearon (1995).

Let us now assume that leaders vary in the extent to which they suffer a subjective loss of utility when devoting their optimal amount of resources towards the war effort. More formally, let $\rho_{i}=\frac{\partial c_{i}}{\partial e_{i}} \forall i \in\{1,2\}$. For simplicity, assume that $\rho_{1}$ is known, while $\rho_{2}$ takes on one of two values, where $L_{2}$ knows the value of $\rho_{2}$ but $L_{1}$ only knows the probability distribution from which it is drawn. That is, assume $\rho_{2}=\bar{\rho}_{2}$ with probability $q$ and $\rho_{2}=\rho_{2}$ with probability $1-q$, where $\underline{\rho}_{2}<\bar{\rho}_{2}$. Then $L_{1}$ knows that with probability $q, L_{2}$ suffers a greater loss of utility, denoted $c_{2}=\bar{c}_{2}$, when selecting the level of war effort necessary to ensure the optimal probability of retaining office, and with probability $1-q, L_{2}$ 's subjective loss of utility is not as great, and $c_{2}=\underline{c}_{2}$, where $\underline{c}_{2}<\bar{c}_{2}$.

There are two perfect Bayesian equilibria to this model, as we demonstrate in the appendix. In one, $L_{1}$ sets $x=p+\underline{c}_{2} \equiv \underline{x}$, which $L_{2}$ accepts regardless of type and thus war never occurs. In the other, $L_{2}$ sets $x=p+\bar{c}_{2} \equiv \bar{x}$, which $L_{2}$ accepts if and only if $c_{2}=\bar{c}_{2}$ and therefore the ex ante probability of war is $1-q$. The former equilibrium obtains when

$$
q \leq \frac{c_{1}+\underline{c}_{2}}{c_{1}+\bar{c}_{2}} \equiv \hat{q}
$$

Simple comparative statics on 1 tell the rest of the story. Recall that $c_{2}=\bar{c}_{2}$ when $\rho_{2}=\bar{\rho}_{2}$, where $\rho_{2}=\frac{\partial c_{2}}{\partial e_{2}}$. Since $\frac{\partial e_{2}^{*}}{\partial W_{2}}>0, c_{2}$ increases as $W_{2}$ increases. Note that the difference between $\bar{c}_{2}$ and $\underline{c}_{2}$ must increase as $W_{2}$ increases. $^{20}$ Therefore, $\hat{q}$ decreases as $W_{2}$ increases, and the conditions sustaining an equilibrium in which there is a non-zero probability of war become easier to satisfy. This indicates that democracies make attractive targets, as others have argued $(\underline{\text { Schultz } 2001})$. Finally, since $\frac{\partial c_{1}}{\partial e_{1}^{*}}>0$ and $\frac{\partial e_{1}^{*}}{\partial W_{1}}>0, c_{1}$ increases as $W_{1}$ increases. Since $\hat{q}$ increases as $c_{1}$ increases, the conditions sustaining the equilibrium with a positive probability of war become more difficult to satisfy as $W_{1}$ increases.

\footnotetext{
${ }^{20}$ This is equivalent to the claim that if $f^{\prime}(x)>g^{\prime}(x)$, then $f(a)-g(a)>f(b)-g(b) \forall a>b$.
} 
This leads to our first key result.

Proposition 1. The equilibrium exhibiting a positive probability of war exists under conditions that are more difficult to satisfy as $W_{1}$ increases or $W_{2}$ decreases.

This result provides little support for the democratic peace as it is conventionally understood. It suggests that we ought to observe a monadic peace in that leaders of democracies must be more confident that their opponent suffers large costs when mobilizing for war before they are willing to make demands that carry a risk of rejection than are leaders of other systems. As Bueno de Mesquita et al. (1999) observe, most studies conclude that there is little evidence of a monadic peace, and they consider the fact that their model does not anticipate such an effect to be a virtue. Further, since it indicates that the conditions under which a leader is willing to make a demand that carries some risk of war are more readily satisfied when the opponent is democratic, the incomplete information version of our model appears to indicate that war is least likely to occur when the challenger is democratic and the target autocratic. However, there is strong evidence that such dyads are amongst the most conflict-prone (Bennett 2006). Perhaps surprisingly, the model also indicates that the most war-prone dyads would consist of an autocratic challenger and a democratic target, which are also amongst the most conflict-prone. Thus, our model suggests that dyads consisting of one democratic and one autocratic state may exhibit either the highest or lowest risk of war, relative to all other combinations, depending upon which is the challenger.

All models are simplifications. We are by no means convinced that the ultimatum protocol perfectly describes the way states actually negotiate. A vast literature based upon this assumption has developed, and similar explanations for war emerge even under richer protocols. $^{21}$ Yet, regardless of whether this protocol perfectly matches real life negotiations, the important point is that, since the democratic peace does not emerge under a commonly analyzed protocol, the core assumptions of selectorate theory cannot be said to unambiguously predict the democratic peace.

\footnotetext{
${ }^{21}$ See Powell (1999), Tarar and Leventoğlu (2008), ?, and Tarar (N.d.).
} 


\section{Individual Strategy and Public Good Provision}

We turn now to the analysis of several closely related variants of a simple model of leader selection. In each, members of the selectorate, denoted $S$, may either retain the incumbent leader, $L$, or replace $L$ with some challenger, $C$.

The only difference between the variants is the decision rule employed by members of $S$. We first solve the game using a decision rule that mirrors that used by Bueno de Mesquita et al. (2003). We then present the results obtained using several additional decision rules. Some of these are less closely related to the core assumptions of selectorate theory but might strike some as worth considering in order to build an alternative theory to arrive at the conclusions offered by Bueno de Mesquita et al. (2003).

Each variant begins with $L$ and $C$ simultaneously stating their platforms. A platform consists of two elements: a bundle of proposed expenditures on the provision of private goods, which we denote $\nu^{j}=\sum_{i}^{S} \nu_{i}^{j} \forall j \in\{L, C\}, i \in S$, and a budget for the provision of public goods, which we denote $\gamma^{j}$. No candidate can propose to spend more than the total amount of resources available, which we treat as fixed and denote $R$. Thus $\nu^{j}+\gamma^{j} \leq R$.

We model the provision of private goods in a straightforward manner, as did Bueno de Mesquita et al. (2003). In order to deliver $\nu_{i}^{j}$ to individual $i$, candidate $j$ expends precisely $\nu_{i}^{j}$ resources. However, we assume a more complicated production technology for public goods. In order to deliver $\mu^{j}$ units of public goods, which by definition will be consumed by all $i \in S$, candidate $j$ must expend $\gamma^{j}$ resources. $^{22}$

We further assume that the production of public goods is inefficient, by which we mean that $\gamma^{j}>\mu^{j}$. That is, when candidate $j$ allocates $\gamma^{j}$ to the production of public goods, the result is only worth $\mu^{j}$ to any given member of $S$. Nonetheless, it may be true that $\sum_{i}^{S} \mu^{j} \geq \gamma^{j}>\mu^{j}$, indicating that the net social value exceeds the cost of production.

\footnotetext{
${ }^{22}$ We might assume the amount of public goods provided is increasing with the resources expended, i.e., $\frac{\partial \mu^{j}}{\partial \gamma^{j}}>0$. We might further assume that the amount of goods produced is increasing at a decreasing rate, or $\frac{\partial^{2} \mu^{j}}{\partial^{2} \gamma^{j}}<0 \forall j \in\{L, C\}$. However, none of the key results depend upon such restrictions.
} 
Note that without assuming $\gamma^{j}>\mu^{j}$, we could not possibly conclude that as $W$ increases, $L$ is more likely to rely upon the provision of public goods in order to retain office, opting for private goods when $W$ is smaller. If $\gamma^{j} \leq \mu^{j}$, there would be no reason for any leader to rely upon the provision of private goods, even if $W$ were equal to 1 .

After $L$ and $C$ state their platforms, the selectorate decides which candidate to support. Throughout, we will assume that platforms are both credible and common knowledge. ${ }^{23} \mathrm{We}$ also assume $L$ 's level of efficiency at generating public goods is common knowledge, though members of $S$ must rely upon a common prior expectation with respect to $C$ 's ability to produce public goods, such that for a given $\gamma^{C}$, all $i$ expect to receive $\hat{\mu}_{\gamma^{C}}^{C} \cdot{ }^{24}$

Initially, we assume that members of $S$ care only about the amount of public and private goods they receive, though below, we will allow for expressive utility to be derived from the act of supporting $L$. Yet in every version, $i$ 's value for having $j$ in office is $\nu_{i}^{j}+\mu^{j}$. We assume candidate $j$ receives $R-\nu^{j}-\gamma^{j}$ if they are selected to be leader, and 0 otherwise.

Finally, we assume throughout that current members of $W^{L}$ can expect to remain in $L$ 's coalition if and only if they support $L$, where $W^{j} \subset S$ denotes a set of size $W$ whose elements are non-identical to those in $W^{\neg j} \subset S$, which also contains $W$ members. We also assume that every member of $S$ who supports $C$ has the same probability of being included in $W^{C}$, and thus expect to receive private goods from $C$ only with probability $\frac{W}{S}$, which Bueno de Mesquita et al. (2003) identify as a critical assumption of selectorate theory.

This model is simpler than any of the models discussed in Bueno de Mesquita et al. (2003). For example, we simply assume that $R$ resources are available to $L$ without considering the leader's choice of tax rates or individual decisions with respect to leisure versus labor decisions, etc. While lacking much of the nuance of the models the authors present, this simple setup is sufficient to illustrate our argument.

\footnotetext{
${ }^{23}$ Naturally, one might argue that candidates can very easily choose to allocate fewer resources once in office than they had pledged beforehand. However, since Bueno de Mesquita et al. (2003) assume credible commitments, we will as well.

${ }^{24}$ Bueno de Mesquita et al. (2003, 1999) make a similar assumption, though this is not strictly necessary for any of our results.
} 
Now consider the solution to this game when employing a decision rule mirroring the one used in Bueno de Mesquita et al. (2003). That is, let $L$ retain office so long as $W$ members of $S$ derive greater utility from having $L$ remain in office. More formally, we assume $L$ retains office if $W$ members of $S$ determine that $E U_{i}(L$ wins $) \geq E U_{i}(C$ wins $)$, or

$$
\nu_{i}^{L}+\mu^{L} \geq \frac{W}{S} \nu_{i}^{C}+\hat{\mu}_{\gamma^{C}}^{C}
$$

Suppose $C$ promises to allocate all of $R$ in the form of private goods, giving $\frac{R}{W}$ to each member of $W^{C}$. Then $L$ can secure office either by setting $\nu_{i}^{L}=\frac{R}{S} \forall i \in W^{L}$ or by allocating $\gamma^{L}\left(\frac{R}{S}\right)$, which produces $\mu^{L}=\frac{R}{S}$.

Note that so long as $W \frac{R}{S}>\gamma^{L}\left(\frac{R}{S}\right)$, the former is less cost effective than the latter and $L$ will prefer to secure the support necessary for retaining office by providing public goods rather than private goods. In other words, we can readily identify a perfect Bayesian equilibrium in which $L$ sets $\gamma^{L}=\gamma^{L}\left(\frac{R}{S}\right), \nu^{L}=0, C$ sets $\gamma^{C}=0, \nu_{i}^{C}=\frac{R}{W} \forall i \in W^{C}$ and $\nu_{m}^{C}=0 \forall m \in S / W^{C}$, and $L$ retains office. In words, there exists an equilibrium in which $L$ retains office by providing public goods that match the quantity of private goods that members of the selectorate could expect to receive if $C$ took office. Importantly, this equilibrium is more likely to obtain as $W$ increases or $S$ increases. Thus we have derived some of the most important results of selectorate theory from our model, simple though it is, having used the same decision rule as the authors.

A few words about the decision rule are in order. Inequality 2 focuses on whether $W$ members of $S$ derive greater utility from having $L$ in office than they do $C$. As Bueno de Mesquita et al. (2003) did, we have identified the conditions under which one outcome is preferred to another. However, the problem with employing this decision rule is that it does not link individual strategies to aggregate outcomes. While we have required $L$ and $C$ to choose strategies that maximize their expected utilities, we have not required members of $S$ to do so. In fact, we have not even specified what strategies members of $S$ employ. 
If we assumed that the outcomes that maximize group utility obtain without requiring that each player adopt their individual best response to the other players' best responses, there would be no dilemma to the one-shot Prisoner's Dilemma, one of the most famous applications of non-cooperative game theory. Nonetheless, this is how Bueno de Mesquita et al. (2003) determine whether $L$ retains office.

We must acknowledge that a vibrant literature in political science similarly assumes that outcomes preferred by sufficiently large number of voters will obtain in equilibrium. ${ }^{25}$ Such is the case in spatial models of elections, for example, where it is often assumed that there is full turnout, only two candidates to choose from, a single policy dimension, and each voter votes for whichever candidate has proposed a platform nearest their ideal point.

However, there is a key difference between selectorate theory and the canonical spatial model of voting. In such models, the assumption that voters support the candidate who proposes a platform closest to their ideal point, without regard for whether their individual decision to support that candidate has an appreciable impact on the likely outcome of the election, is of little consequence with respect to the substantive conclusions. That is, while this assumption abstracts away from the important question of why voters who are solely concerned with policy outcomes choose to vote at all, the assumption of sincere voting in a full turnout election with two candidates does not distort our analysis of what platforms the candidates will propose, which is typically what scholars seek to identify with such models. As with any formal model, it is not particularly useful to observe that one of the key assumptions is unlikely to be empirically tenable without further demonstrating that without this assumption, the results would differ in substantively important ways.

In other words, the fact that Bueno de Mesquita et al. (2003) do not require individual voters to choose strategies that maximize their expected utility would of course not be a problem if the substantive conclusions following from their model would not differ if we required voters to do so. If a model in which members of $S$ are required to choose strategies

\footnotetext{
${ }^{25}$ For overviews of the literature, see, inter alia, McCarty and Meirowitz (2007) and Mueller (2003).
} 
that maximize their individual expected utilities, and where aggregate outcomes reflect these choices, continued to suggest that $L$ is more likely to provide public goods as $W$ increases and $S$ decreases, the authors' choice of decision rule would be innocuous.

Unfortunately, that is not the case. Suppose that we no longer assume $L$ retains office when $W$ members of $S$ derive greater utility from having $L$ remain in office, but instead require each $i \in S$ to adopt the individual strategy that maximizes their expected utility. More formally, let each $i \in S$ support $L$ if and only if $E U_{i}($ support $L) \geq E U_{i}($ support $C)$, or

$$
\bar{p}\left(\nu_{i}^{L}+\mu^{L}\right)+(1-\bar{p}) \hat{\mu}_{\gamma^{C}}^{C} \geq \underline{p} \mu^{L}+(1-\underline{p})\left(\frac{W}{S} \nu_{i}^{C}+\hat{\mu}_{\gamma^{C}}^{C}\right)
$$

where $\bar{p}$ denotes the probability that $L$ retains office with $i$ 's support, $\underline{p}$ denotes the probability that $L$ retains office absent $i$ 's support. Given the nature of public goods, we argue that this is a more appropriate decision rule. If each $i$ chooses whom to support by evaluating inequality $3, i$ will have assumed that she will receive any public goods provided by $j$ regardless of whether she supports $j$, yet also assumed that she must support $j$ in order to receive private goods from $j$ in the event that $j$ takes office.

This brings our to our next key result.

Proposition 2. There exists a perfect Bayesian equilibrium to the leader selection game using the decision rule outlined by inequality 3, where $L$ sets $\nu^{L}=\gamma^{L}=0$.

The intuition behind this result is that when members of $S$ concern themselves solely with the amount of goods they expect to receive as a function of whom they individually choose to support, if $L$ does not propose to allocate any resources to anyone, every member of $S$ may nonetheless be indifferent between supporting $L$ and supporting $C$. If we assume that members of $S$ default to supporting $L$ when indifferent, as we implicitly did above, then $L$ wins despite not allocating any goods to anyone because no individual member of $S$ can benefit by deviating and pledging their support to $C$. If they $\operatorname{did}$ so, $C$ still would not win, and they would continue to expect to receive nothing. 
This equilibrium pretty clearly does not match reality, though its existence is telling theoretically. Of course, it rests critically upon an arbitrary assumption about how members of $S$ behave when indifferent. So let us now allow members of $S$ to receive arbitrarily small rewards or punishments for expressing support for the incumbent, irrespective of the outcome. These parameters will function primarily to break ties. More formally, let each $i \in S$ receive $\alpha_{i}^{L} \sim U[-\epsilon, \epsilon]$ when supporting $L$. Let each $\alpha_{i}^{L}$ be known by $i$ and only $i$. That is, no member of $S$ can know how non-material considerations will influence the behavior of any other member of $S$, nor can the candidates. The primary effect of these terms is to make selector's decisions probabilistic, preventing strange equilibria like the one just discussed. We then obtain the next key result.

Proposition 3. In any perfect Bayesian equilibrium to the leader selection game with nonmaterial incentives to support the incumbent, $L$ 's choice of $\gamma^{L}$ is increasing in $W$, all else equal, but only takes on positive values if the difference between $\bar{p}$ and $p$ is non-trivial.

This appears to support the conclusions of Bueno de Mesquita et al. (2003). All else equal, $L$ allocates greater resources towards the provision of public goods as $W$ increases. If we associate large values of $W$ with democracy, this would seem to indicate that democrats are more likely to provide public goods than are their authoritarian counterparts.

However, the restriction that $L$ only sets $\gamma^{L}>0$ when $\bar{p}-\underline{p}$ takes on non-trivial values is quite powerful. Scholars have long recognized that it is highly implausible that a single voter's decision influences the outcome of a national election in a modern electoral democracy. ${ }^{26}$ If the best explanation for the provision of public goods we can come up with is that voters in democracies are dissuaded from supporting the challenger for fear that if they do not support the incumbent, they significant decrease the prospects of the leader being retained and therefore being able to provide the public goods they pledged, we do not have much of an explanation of the supposed link between democracy and public goods provision at all.

\footnotetext{
${ }^{26}$ Riker and Ordeshook (1968) sought to resolve the paradox that voters do in fact vote in elections even though they cannot hope to influence the outcome. While many others have offered alternative explanations, very few have disputed the claim that voters are unlikely to believe that their vote has a non-trivial impact.
} 
For this reason, we now consider two additional variants of the model. These are less closely related to the core assumptions of selectorate theory. Yet, if we could arrive at essentially the same conclusions as Bueno de Mesquita et al. (2003) after relaxing the strong assumption of extreme self-interest, many might feel that it is of little interest that selectorate theory does not itself produce such conclusions. After all, the most recent literature suggests voters care about how the outcome of the election affect the nation as a whole. ${ }^{27}$

We thus replace $\alpha_{i}^{L}$ with $\alpha_{i}^{L}\left(\mu^{L}\right)$, where we assume $\frac{\partial \alpha_{i}^{L}\left(\mu^{L}\right)}{\partial \mu^{L}} \geq 0 \forall i \in S$. That is, we assume that that all members of the selectorate derive utility from expressing support for leaders who they expect will produce goods that benefit the entire society. ${ }^{28}$

Proposition 4. If expressive utilities are sufficiently sensitive to public good provision, $L$ sets $\gamma^{L}>0$ and the relationship between democracy and $L$ 's choice of $\gamma^{L}$ is ambiguous.

When the level of support an incumbent leader expects to receive is directly related to the amount of public goods they produce, not because members of $S$ must support $L$ to receive the goods but because they derive greater utility from supporting $L$ for expressive reasons when $L$ produces public goods, all leaders produce public goods. Whether democrats produce more is unclear.

We could of course assume that it is only in some societies that members of $S$ derive expressive utility from supporting leaders who produce public goods. Such an argument would seemingly allow us to account for the results reported by Bueno de Mesquita et al. (2003). We hope the problems with such an argument are self-evident. Not only would such an argument border on tautological, but it would require us to explain the difference in standard of living between that in the Western world and less developed and less democratic areas, such as Sub-Saharan Africa, by assuming that it is only in the Western world that people worry about the wellbeing of their fellow citizens.

\footnotetext{
${ }^{27}$ See in particular Edlin, Gelman and Kaplan (2007), Fowler (2006), Grafstein (2009), and especially Feddersen, Gailmard and Sandroni (2009).

${ }^{28}$ We might alternatively assume that the expressive utility is a function of the difference between $\mu^{L}$ and $\hat{\mu}_{\gamma^{C}}^{C}$. However, this is would have little bearing on our substantive conclusions. Further, there appears to be evidence that voters do in fact vote based on blind retrospective evaluations (Achen and Bartles N.d.).
} 
Finally, we consider one last variant of the model. Here we assume that in all societies, there are two general types of selectors. All members of $S$ derive utility from the goods provided to them by the winner of the selection contest, and all members of $S$ consider the impact of their individual decision of whom to support on the likely outcome. Further, we continue to assume that all members of $S$ derive some arbitrarily small expressive utility from supporting $L$. The only difference is that, for some members of $S$, this utility is independent of $\mu^{L}$, while for others it is not. That is, for one group, $\frac{\partial \alpha_{i}^{L}}{\partial \mu^{L}}=0$, while for some group $P \subset S, \frac{\partial \alpha_{p}^{L}\left(\mu^{L}\right)}{\partial \mu^{L}} \geq 0 \forall p \in P, p \neq i$. We refer to $P$ as the set of $S$ that is public-spirited.

Proposition 5. Provided the proportion of $S$ that is public-spirited is sufficiently large, it is impossible for $L$ to retain office without $\gamma^{L}>0$ after $W$ crosses some threshold while it remains possible to set $\gamma^{L}=0$ for values of $W$ below this threshold.

This result would appear to bring us full circle back to the conclusions of Bueno de Mesquita et al. (2003). However, if we are comfortable trying to explain behavior by assuming actors derive inherent utility from their actions, even if our results would not hold without such assumptions, we should also assume that $L$ derives inherent utility from setting $\gamma^{L}>0$.

Corrolary 1. If leaders derive sufficient utility from providing for all of their people, there is an equilibrium in which the choice of $\gamma^{L}$ is independent of $W$.

We believe there is little value to the argument that good outcomes occur in democracies because most people demand good things, while outcomes under autocracy are typically bad because autocratic leaders are typically too selfish to want to help their people. Important elements of this argument border on tautological. But for the fact that many scholars strongly believe that democracy itself is a cause of good outcomes, it is difficult to imagine that an argument such as this would be met with anything but extreme skepticism. ${ }^{29}$

\footnotetext{
${ }^{29}$ For example, it is unclear how this argument differs substantially from the claim that suicide terrorists behave as they do because they derive inherent utility from their actions, irrespective of the obvious material incentive to behave otherwise, or the claim that voters vote because it is their civic duty to do so and they derive expressive utility from doing their duty (Riker and Ordeshook 1968), an explanation that failed to convince many that the paradox of voting had been resolved.
} 


\section{Do Democracies Actually Provide More Public Goods?}

The primary reason we are reluctant to embrace the results above, though they appear to account for the empirical results reported by Bueno de Mesquita et al. (2003), is that we believe those results tell us relatively little about the relationship between democracy and the provision of public goods. Specifically, we see good reason to question whether there is in fact compelling evidence of a relationship between regime type and the provision of true public goods, and so we are hesitant to introduce assumptions about the desire to produce public goods, or to see public goods produced, on behalf of leaders or the selectorate.

Our skepticism of the claim that democracies produce public goods is twofold. First, we see little reason to conclude that many of the outcomes analyzed by Bueno de Mesquita et al. (2003), such as the number of hospital beds, rates of female secondary education, immunizations, and so forth, are either non-rival or non-excludable. While it may be true in practice that few governments restrict access to hospitals or schools on the basis of political allegiance, there is an important difference between policies that exhibit some level of publicspiritedness and proper public goods. It is eminently feasible, for example, to provide a hospital bed to one citizen without all others benefiting simultaneously.

This distinction is important because Bueno de Mesquita et al. (2003) never argue that any leader, regardless of the size of $W$, provides public goods because they wish to benefit everyone. Rather, their key claim is that leaders who desire nothing more than to secure the support of the minimally necessary number of selectors will find it economically efficient to provide goods that satisfy everyone by virtue of the fact that they are non-excludable. Thus, it is absolutely central to the logic of their argument that leaders of large $W$ systems provide true public goods, goods that benefit everyone, even though democratic leaders, like all leaders, have no particular desire to make everyone happy.

This brings us to our second source of skepticism. If leaders of democracies, due to the size of $W$, had no choice but to implement policies that benefit everyone, there should be unambiguous evidence that democracy is good for the poor and for the environment. 
We leave aside concerns that even with respect to health and education outcomes, the evidence is not as clear as Bueno de Mesquita et al. (2003) suggest (Nelson 2007) and grant, for the sake of argument, that more people receive more goods in democracies than nondemocracies. But if democratic governments do in fact provide true public goods, we should expect that the poorest in society, who presumably are not part of $W$, will only benefit from goods provided to all. We also should see clear evidence that democracy is associated with greater environmental stewardship, since clean air and preservation of the environment are canonical, textbook examples of true public goods, whose provision is non-excludable.

But the evidence that democracy reduces poverty is not unambiguous. Ross (2006) argues that the apparent relationship between democracy and poverty reduction (e.g., Przeworski et al. (2000)) is misleading. Most studies of the relationship between democracy and poverty, often proxied by infant mortality rates, rely upon significantly reduced samples due to missing data. If the data were missing randomly, this would naturally be of little concern. Unfortunately, as Ross demonstrates quite compellingly, this is not the case.

Ross hypothesizes that democracies can generally be expected to report infant mortality rates, in addition to a broad array of other statistics. Yet the incentive for autocrats to do so is not apparent. That is, unless there is outside pressure on them to do so, which is likely to be the case for the least economically developed autocracies, who frequently require assistance from the IMF. The IMF, which maintains a database on statistics related to economic development, expects clients to report these statistics. Ross tests this hypothesis and finds that there are less likely to be missing values for infant and child mortality data for both democracies and states bound by IMF agreements, and that, amongst autocracies, there is a strong postive relationship between economic development and missing data. Therefore, an analysis of regime type and infant mortality that does not correct for the non-random nature of the missing data will be biased in favor of finding that autocracies perform worse.

Ross then uses multiple imputation to correct the problem and finds that there is no relationship between democracy and infant or child mortality. This should not surprise us, 
because the policy tools available to governments to reduce infant and child mortality are not technically public goods and can be targeted to some parts of the population and not others. Finally, he demonstrates that there is also no relationship between regime type and the ratio of treatments for various maladies received by rich relative to the poor.

Similarly, Scruggs (N.d.) argues that most of the work purporting to show that democracy reduces environmental degradation (e.g., Li and Reuveny (2006)) suffers from serious shortcomings, such as reducing environmental degradation to one or two measures, such as sulfur dioxide emissions or deforestation. Offering a systematic evaluation of 58 studies on the topic, Scruggs (N.d.) seeks to correct common flaws. Most notably, he employs eight indicators to provide a broader measure of environmental degradation, and also assess the impact of changes in regime type on the trajectory of environmental performance, rather than simply analyzing contemporaneous levels of both, thereby addressing concerns that economic development might cause both democracy and cleaner environments. After addressing these concerns, Scruggs (N.d.) finds no evidence that democracy promotes better stewardship of the environment. He concludes that economic development is more likely responsible for the apparent association.

Admittedly, a cursory review of a few recent studies cannot settle the debate. We acknowledge this. Moreover, we stress that we do not dispute that our reformulation of selectorate theory predicts that the resources at a nation's disposal are more likely to be shared broadly in democracies than non-democracies. The distinction between providing benefits to many as opposed to all is not inconsequential, but we do not wish to exaggerate it either. Depending upon the question of interest, scholars sometimes will, and sometimes will not, find the distinction between our preferred interpretation of selectorate theory and the claims of Bueno de Mesquita et al. (2003) to be relevant. ${ }^{30}$

\footnotetext{
${ }^{30}$ For example, several scholars find that democracy does not decrease the risk of civil war (Fearon and Laitin 2003, Henderson 2002), though see also Hegre (2001). Insofar as one of the explanations for civil war is popular grievances (Collier and Hoeffler 2004), the question of whether democrats produce policies that benefit everyone rather than $\frac{W}{N}$ of the people, may be quite important.
} 


\section{Conclusion}

Selectorate theory has proven to be very influential in the study of politics, not only because it claims to provide an explanation for the democratic peace, but also to yield important insights into international cooperation, and a variety of domestic outcomes. Many also find the theory attractive due its relative parsimony. Seemingly, we need only assume that leaders typically enjoy wielding power and that their subjects prefer to receive more goods rather than less, in order to explain a wide range of empirical regularities. Even those who are reluctant to claim that democracies instill in their citizens greater virtues can nonetheless believe that democratic governance is critical for producing peace (at least amongst fellow democracies) and prosperity (assumed to be more likely to obtain when leaders provide public goods, such as the rule of law). The appeal of such a theory is obvious. The implications are both surprising, given the nature of the assumptions, as well as normatively satisfying.

Yet, as we hope to have illustrated, this stylized interpretation is not warranted. Specifically, some of the most celebrated results reported in Bueno de Mesquita et al. (1999) and Bueno de Mesquita et al. (2003) are less parsimonious than the authors claim, as they depend critically upon modeling assumptions that neither are justified by the core logic of their theory nor substantively easy to defend.

With respect to the democratic peace, Bueno de Mesquita et al. (1999) implicitly assumed that when states negotiate with one another, neither of them has any influence on the distribution of benefits each would receive. Moreover, they assumed that peace requires the consent of only one party, in contrast to the traditional interpretation of anarchy. We analyzed a very simple model, one in which we assumed the very patterns Bueno de Mesquita et al. (1999) derived from their model with respect to the relationship between domestic institutions and optimal war effort. Despite this substantial simplification, we found that the democratic peace obtained under their bargaining protocol, but appears incompatible with the more flexible protocol found in Fearon (1995), which has subsequently become one of the standard ways of modeling crisis bargaining. 
We acknowledge that the particular manner in which we chose to represent incomplete information does not follow directly any of the assumptions in Bueno de Mesquita et al. (1999), who assumed complete information. Yet even if the democratic peace could be reconciled with our model using a different approach to modeling incomplete information, we would continue to argue that there is no unambiguous evidence that the core assumptions of selectorate theory point towards the democratic peace. We contend that if a theory can only account for a given finding under arbitrary specifications that are not informed by the core assumptions of the theory, that theory provides a poor explanation for said finding.

After replacing the non-standard bargaining protocol used by Bueno de Mesquita et al. (1999) with the ultimatum bargaining protocol, we derived rather puzzling results regarding the relationship between regime type and conflict. We are relatively untroubled by these results because we see the model we presented as being useful for illustrating the fragile nature of the results reported by Bueno de Mesquita et al. (1999) but not necessarily so for generating novel empirical implications. For the sake of argument, we found it useful to assume from the outset that certain patterns with respect to war effort. However, it is not obvious to us, given our subsequent arguments about public goods, that we would want to build a model on such assumptions if we wished to generate empirical implications.

With respect to public goods, though they are non-excludable by definition, in order to prove that leaders of large $W$ systems are required to provide public goods if they wish to retain office, the authors assumed that members of $S$ make decisions without regard for the relationship between their individual behavior and their individual access to goods. Members of $S$ are fundamentally non-strategic in their models.

To illustrate this, we analyzed a very simple model, one that lacked many of the moving parts found in Bueno de Mesquita et al. (2003). Despite these differences, we first showed that if we employed the same decision rule as the authors, we could reproduce their key claims. However, once we adopted more appropriate decision rules, we no longer found evidence that democrats are likely to distribute public goods. 
In some respects, our argument is anything but novel, though its implications may surprise some. Scholars have long recognized it is important to focus on the incentives facing individual members of the (s)electorate. We also consider it to be reassuring that Smith and Bueno de Mesquita (N.d.) have developed a more sophisticated model than the simple one we analyzed here, which they use to explain the paradox of voting. Their model, like ours, suggests that members of large electorates do not choose whom to support on the basis of which candidate proposes to advance the public interest, but rather base their decisions upon how their individual behavior impacts their access to particularistic goods.

We also demonstrated that if we assume members of $S$, or even leaders themselves, are less narrowly self-interested than they are assumed to be in selectorate theory, we might expect to observe public goods provision, and potentially a relationship between regime type and public goods provision. However, the models that allowed for this had some unappealing properties. We are reluctant to read too much into these results.

There are important questions to be raised about the extent to which the policies enacted by democratic governments produce benefits that are non-rival and non-excludable - which is, after all, the definition of a public good. While the policies of modern democracies may exhibit some level of public spiritedness, that is not the same as providing a public good. ${ }^{31}$ The important distinction is whether leaders of democracies produce value primarily for the members of their winning coalition, which by definition already entails providing greater services than we would expect to observe in autocracies, or serve the interests of everyone in society. The analysis presented in Bueno de Mesquita et al. (2003) cannot address this question adequately. Recent articles that investigate the relationship between regime type and poverty, on the one hand, and regime type and environmental degradation, on the other, find little evidence that democracy does helps the poor (Ross 2006) or discourages environmental degradation (Scruggs N.d.).

\footnotetext{
${ }^{31}$ This is to say nothing of the question of whether the bulk of government spending does in fact improve the welfare of the average citizen. Suffice it to say that substantial literatures in public choice and economics challenge this view (Mueller 2003).
} 
We leave it for others to evaluate whether economic factors or other forces are more proximately responsible for the outcomes we often attribute to democracy. While the democratic peace is widely accepted, we are not the first to observe that the historical record simply has not provided us sufficient variation to allow us to confidently rule out all the alternative explanations for the dramatic reduction of militarized conflict in the Western world since 1945. ${ }^{32}$ Insofar as one of the primary explanations for the democratic peace, selectorate theory, yielded many additional observable implications that were empirically corroborated, one might not be troubled by this. Yet we believe that our results, in combination with those of Clarke and Stone (2008) and others, largely undercut any such argument. Many of the things that ought to be true if the common interpretation of selectorate theory was correct are, at minimum, open to dispute.

Note that our criticisms deal specifically with how Bueno de Mesquita et al. (2003, 1999) characterize the behavior of leaders of democratic polities, which has played a deeply influential role in the study of the democratic peace. However, one of the central motivating puzzles discussed in Bueno de Mesquita et al. (2003) concerns the empirical observation that those leaders whose people suffer most appear to have the strongest hold on power. Their answer to this important puzzle is that, in authoritarian regimes, "good policy is bad politics". Nothing in the variants of the model that continue to assume self-interested behavior challenges this claim, nor do we wish to suggest this is not a compelling, if unhappy, explanation. Though we did not focus on this question above, the version of our model with expressive utilities that are not tied to the leader's provision of public goods indicates that the probability that a leader remains in office is strictly decreasing with the size of the winning coalition, and that the rents from office follow a similar pattern. Thus, we agree with Bueno de Mesquita et al. (2003) that the leaders of less inclusive regimes will provide little for most of their people while extracting vast resources for personal consumption, and nonetheless will be relatively secure with respect to their hold on office. ${ }^{33}$

\footnotetext{
${ }^{32}$ See Gartzke (2007), Gibler (2007), Henderson (2002, 2009) and Ward, Siverson and Cao (2007).

${ }^{33}$ See the proof of Proposition 3 in the appendix.
} 
But we see no reason to stop there. While we acknowledge that it is an oversimplification, we argue that all leaders govern primarily with the interests of the members of their winning coalition in mind, with little regard for the well-being of anyone else. To be sure, there are problems with this claim. Leaders of democracies probably rely more on club goods than either private or public goods. ${ }^{34}$ They also often implement policies that will disproportionately benefit groups that, in aggregate, support them at higher rates than other groups, rather than explicitly targeting resources towards specific individuals whom they know have expressed support for them. We readily acknowledge these shortcomings. Yet insofar as there is a lot to be gained from broad generalizations, and we believe there is, we find this simplified claim more useful than the equally simplified claim that autocrats serve the interests of their winning coalitions while democrats serve the interests of everyone.

Our criticism of the depiction of democratic politics in Bueno de Mesquita et al. (2003, 1999), where leaders have no choice but to make everyone happy, where there is no real meaningful distributive element to the political process, should not be interpreted as indicating that we find no merit in selectorate theory. We simply believe that some of the most widely celebrated claims associated with selectorate theory, specifically with regards to the democratic peace and the provision of public goods, are problematic. Nonetheless, we continue to believe that the core assumptions that leaders desire to remain in office, and that important political outcomes can be better understood if we focus on variation across polities in terms of the proportion of the population that has a say in who gets to be leader and the subset of that group whose support is minimally necessary, are profoundly useful.

\footnotetext{
${ }^{34}$ We thank Erik Gartzke for pointing this out.
} 


\section{Appendix}

\section{Crisis Bargaining}

Proposition 1. There are two pure strategy PBEs to the model with incomplete information. In the first, $L_{1}$ sets $x=\underline{x}$; the strong $L_{2}$ accepts if and only if $x \leq \underline{x}$; the weak $L_{2}$ accepts if and only if $x \leq \bar{x}$. In the second, $L_{1}$ sets $x=\bar{x}$; the strong $L_{2}$ accepts if and only if $x \leq \underline{x}$; the weak $L_{2}$ accepts if and only if $x \leq \bar{x}$.

$L_{1}$ 's optimal offer is straightforward. If $L_{1}$ sets $x>\bar{x}$, war results with certainty, which cannot be optimal since $\bar{x} \equiv p+\bar{c}_{2}>p-c_{1}$. If $L_{1}$ sets $x<\underline{x}, L_{1}$ foregoes better terms of agreement than would have been available had $L_{1}$ set $x=\underline{x}$, which $L_{2}$ is certain to accept regardless of type. If $L_{1}$ sets $\underline{x}<x<\bar{x}, L_{1}$ receives the same payoff when $L_{2}$ is strong as $L_{1}$ would had received had $L_{1}$ set $x=\bar{x}$, and $L_{1}$ 's payoff from having the weak $L_{2}$ accept is strictly inferior to $L_{1}$ 's payoff from having the weak $L_{2}$ accept $x=\bar{x}$. Given the choice between $x=\underline{x}$ and $x=\bar{x}, L_{1}$ 's strategy follows immediately from inequality 1 .

$L_{2}$ 's strategy follows readily from setting $u_{L_{2}}($ acc $) \geq E U_{L_{2}}(r e j) \Leftrightarrow 1-x \geq 1-p-c_{2}$. When $L_{2}$ is strong, i.e. $\rho=\underline{\rho}$ and thus $c_{2}=\underline{c}_{2}$, this simplifies to $x \leq p+\underline{c}_{2} \equiv \underline{x}$. When $L_{2}$ is weak, i.e. $\rho=\bar{\rho}$ and thus $c_{2}=\bar{c}_{2}$, this simplifies to $x \leq p+\bar{c}_{2} \equiv \bar{x}$.

The probability of war in the equilibrium where $L_{1}$ sets $x=\underline{x}$ is 0 . When $L_{1} \operatorname{sets} x=\bar{x}$, the ex ante probability of war is $1-q$. The former equilibrium exists if and only if $q \leq \hat{q}$, the latter if and only if $q>\hat{q}$. Therefore, Proposition 1 follows from the comparative statics on inequality 1 , which are discussed in the text.

\section{Leader Selection}

Proposition 2. The following beliefs and pure strategies constitute a perfect Bayesian equilibrium. $L$ sets $\nu^{L}=0$ and $\gamma^{L}=0 ; C$ sets $\nu_{i}^{C}=\frac{R}{W} \forall i \in W^{C}, \nu_{m}^{C}=0 \forall m \in S / W^{C}$ and $\gamma^{C}=0$; all $i \in S$ support $L$ iff inequality 3 holds, supporting $C$ otherwise, and believe $\hat{\mu}_{\gamma^{C}}^{C}>0$ if $\gamma^{C}>0, \hat{\mu}_{\gamma^{C}}^{C}=0$ otherwise, and believe $\bar{p}=1, \underline{p}=1$. 
Note that the appropriate solution concept is perfect Bayesian equilibrium since the actor's strategies depend upon their beliefs, $\hat{\mu}^{C}, \bar{p}$ and $\underline{p}$. However, note that since the behavior of the selectorate is deterministic, all $i \in S$ can update their beliefs $\bar{p}$ and $\underline{p}$ to certainty. Also note that the actors do not have the opportunity to learn about $\hat{\mu}^{C}$.

Specifically, since every member of $S$ supports $L$ in equilibrium, and $L$ requires the support of only $W$ members of $S$, then from the perspective of any given $i, L$ wins either with or without $i$ 's support. Thus $\bar{p}=1$ and $\underline{p}=1$.

Substituting the equilibrium platforms for $L$ and $C$ into inequality 3 , we get

$$
\bar{p} \cdot 0+(1-\bar{p}) \cdot 0 \geq \underline{p} \cdot 0+(1-\underline{p})\left(\frac{W}{S} \frac{R}{W}\right)
$$

which, after substituting in $\bar{p}=1$ and $\underline{p}=1$, simplifies to

$$
0+0 \geq 0+0\left(\frac{R}{S}\right)
$$

or simply $0 \geq 0$. Thus, $i$ 's strategy is incentive compatible, as $0 \geq 0$ is true by definition.

Given that $L$ expects to retain office, $L$ has no incentive to set $\nu^{L}>0$ or $\gamma^{L}>0$. Thus, $L$ 's strategy is incentive compatible.

$C$ has no incentive to deviate from the equilibrium strategy, since $C$ cannot prevent $L$ from winning by changing her platform, nor does $C$ 's payoff depend upon her platform in any equilibrium in which $C$ does not take office. This completes the proof.

Proposition 3. In any perfect Bayesian equilibrium, each $i \in S$ supports $L$ if and only if

$$
\bar{p}\left(\nu_{i}^{L}+\mu^{L}\right)+(1-\bar{p}) \hat{\mu}_{\gamma^{C}}^{C}+\alpha_{i}^{L} \geq \underline{p} \mu^{L}+(1-\underline{p})\left(\frac{W}{S} \nu_{i}^{C}+\hat{\mu}_{\gamma^{C}}^{C}\right)
$$

where $\hat{\mu}_{\gamma^{C}}^{C}>0$ if $\gamma^{C}>0$ and $\hat{\mu}_{\gamma^{C}}^{C}=0$ otherwise.

Solving inequality 4 for $\alpha_{i}^{L}$, we can alternatively say that $i$ supports $L$ if $\alpha_{i}^{L} \geq \hat{\alpha}_{i}^{L}$, where $\hat{\alpha}_{i}^{L} \equiv\left(\hat{\mu}_{\gamma}^{C}-\mu^{L}\right)(\bar{p}-\underline{p})+(1-\underline{p}) \frac{W}{S} \nu_{i}^{C}-\bar{p} \nu_{i}^{L}$. While candidate $j$ cannot know whether 
$m \in S, m \neq i$ will support $L$, given that $i$ does not know the value of $\alpha_{m}^{L}, i$ knows that the probability that $m \in S, m \neq i$ supports $L$, which we'll denote $S_{m}^{L}$, is given by

$$
S_{m}^{L}= \begin{cases}\frac{\epsilon-\hat{\alpha}_{m}^{L}}{2 \epsilon} & \text { if }-\epsilon \leq \hat{\alpha}_{m}^{L} \leq \epsilon \\ 0 & \text { if } \hat{\alpha}_{m}^{L}>\epsilon \\ 1 & \text { if } \hat{\alpha}_{m}^{L}<-\epsilon\end{cases}
$$

Then $\bar{p}$ is the probability that $L$ 's expected level of support is at least as large as $W$, where $L$ 's expected level of support is $1+\sum_{m} S_{m}^{L}$. Similarly, $\underline{p}$ is the probability that $L$ 's expected level of support without $i$ is at least as large as $W$, or $\operatorname{pr}\left(\sum_{m} S_{m}^{L}\right) \geq W$.

From the perspective of $L$, the probability of retaining office is $\operatorname{pr}\left(\sum_{i}^{S} S_{i}^{L} \geq W\right) \equiv \hat{p}$, and the optimal values of $\nu^{L}$ and $\gamma^{L}$ depend upon $L$ 's expected utility, $\hat{p}\left(R-\nu^{L}-\gamma^{L}\right)$, subject to the constraint $\nu^{L}+\gamma^{L} \leq R$. The optimal allocations can be found by maximizing $\hat{p}\left(R-\nu^{L}-\gamma^{L}\right)-\lambda\left(\nu^{L}+\gamma^{L}-R\right)$ with respect to $\nu^{L}$ and $\gamma^{L}$, respectively.

We are particularly interested in $L$ 's choice of $\gamma^{L}$, which is given by

$$
\lambda=\frac{\partial \hat{p}}{\partial \gamma^{L}}\left(R-\nu^{L}-\gamma^{L}\right)-\hat{p}
$$

Intuitively, this tells us that if $R$ increased, $L$ would increase $\gamma^{L}$ in proportion to the impact of $\gamma^{L}$ on her probability of retaining office, weighted by the rents $L$ extracts from office upon victory, while decreasing $\gamma^{L}$ as her expected probability of retaining office increases.

Clearly, $\hat{p}$ at least weakly decreases as $W$ increases, since $\hat{p}=\operatorname{pr}\left(\sum_{i}^{S} S_{i}^{L} \geq W\right)$. This indicates that, all else equal, $L$ 's optimal $\gamma^{L}$ increases as $W$ increases.

However, inspection of $\hat{\alpha}_{i}^{L}$ clearly indicates that the impact of $\gamma^{L}$ on the probability that any given $i$ supports $L$, or $S_{i}^{L}$, goes to 0 as $\bar{p}-\underline{p}$ goes to 0 . In the limit, as $\bar{p}-\underline{p} \rightarrow 0$, $\frac{\partial \hat{p}}{\partial \gamma^{L}} \rightarrow 0$, and by 5 , we can see that $L$ 's optimal allocation of resources towards the provision of public goods, which cannot take on negative values, must also go to 0 . 
Proposition 4. Now suppose that in any PBE, each $i \in S$ supports $L$ if and only

$$
\bar{p}\left(\nu_{i}^{L}+\mu^{L}\right)+(1-\bar{p}) \hat{\mu}_{\gamma}^{C}+\alpha_{i}^{L}\left(\mu^{L}\right) \geq \underline{p} \mu^{L}+(1-\underline{p})\left(\frac{W}{S} \nu_{i}^{C}+\hat{\mu}_{\gamma}^{C}\right),
$$

where $\hat{\mu}_{\gamma^{C}}^{C}>0$ if $\gamma^{C}>0$ and $\hat{\mu}_{\gamma^{C}}^{C}=0$ otherwise, and $\frac{\partial \alpha_{i}^{L}\left(\mu^{L}\right)}{\partial \mu^{L}} \geq 0$. Unlike above, we no longer constrain $\alpha_{i}^{L}\left(\mu^{L}\right)$ to be distributed uniformly between $[-\epsilon, \epsilon]$.

Solving inequality 6 for $\alpha_{i}^{L}\left(\mu^{L}\right)$, we can alternatively say that $i$ supports $L$ if $\alpha_{i}^{L}\left(\mu^{L}\right) \geq$ $\hat{\alpha}_{i}^{L}\left(\mu^{L}\right) \equiv\left(\hat{\mu}_{\gamma}^{C}-\mu^{L}\right)(\bar{p}-\underline{p})+(1-\underline{p}) \frac{W}{S} \nu_{i}^{c}-\bar{p} \nu_{i}^{L}$.

As above, the impact of $\gamma^{L}$ on $\hat{\alpha}_{i}^{L}\left(\mu^{L}\right) \rightarrow 0$ as $\bar{p}-\underline{p} \rightarrow 0$. However, since $\alpha_{i}^{L}\left(\mu^{L}\right)$ increases with $\mu^{L}$ and $\mu^{L}$ increases with $\gamma^{L}$, we cannot conclude that the probability that $i$ supports $L, S_{i}^{L}$, becomes independent of $\gamma^{L}$ even as $\bar{p}-\underline{p} \rightarrow 0$. Rather, $\frac{\partial S_{i}^{L}}{\partial \gamma^{L}} \geq 0 \forall i \in S$ and $\frac{\partial \hat{p}}{\partial \gamma^{L}} \geq 0$ irrespective of the size of $W$ or $S$. Recall that $L$ 's optimal $\gamma^{L}$, which is still given by 5 , is increasing in $\frac{\partial \hat{p}}{\partial \gamma^{L}}$. Thus, even when $\bar{p}-\underline{p} \rightarrow 0$, the optimal $\gamma^{L}$ remains positive.

Whether $L$ 's optimal $\gamma^{L}$ increases or decreases with $W$ and $S$ is unclear. On the one hand, as $W$ increases, all else equal, $\hat{p}$ decreases, which increases the optimal $\gamma^{L}$. On the other hand, as $S$ increases, $\hat{\alpha}_{i}^{L}\left(\mu^{L}\right)$ decreases, increasing the probability that $\alpha_{i}^{L}\left(\mu^{L}\right) \geq \hat{\alpha}_{i}^{L}\left(\mu^{L}\right)$ holds, meaning $S_{i}^{L}$ increases and $\hat{p}$ increases, which decreases the optimal $\gamma^{L}$. Since democracy is associated with large $W$ and large $S$, this may or may not indicate a greater optimal $\gamma^{L}$ than would obtain for regimes with a small $W$ and a small $S$.

Proposition 5. Let $\frac{\partial \alpha_{p}^{L}\left(\mu^{L}\right)}{\partial \mu^{L}}=0 \forall p \in P \subset S$ while $\frac{\partial \alpha_{i}^{L}}{\partial \mu^{L}}=0 \forall i \in S / P$. Take some arbitrary $W^{D}$ and $W^{A}$ where $W^{D}>W^{A}$. Now fix $S$ to be sufficiently large that $\bar{p}-\underline{p}$ is arbitrarily close to 0 . Then provided $S-P<W^{A}<P<W^{D}$, if follows from the proofs of Propositions 3 and 4 that the optimal $\gamma^{L}$ for the leader must be positive if $W=W^{D}$. However it can be optimal for $L$ to garner the support of $W^{A}$ members of $S / P$ while setting $\gamma^{L}=0$. Note that the first expression in $L$ 's optimal $\gamma^{L}$, given by 5 , is arbitrarily close to 0 when $\bar{p}-\underline{p}$ is arbitrarily close to 0 , as we have already stipulated. Further, $\hat{p}$ increases as $W^{D}$ decreases, decreasing the optimal $\gamma^{L}$ until it eventually reaches 0 . 
Corrolary 1. Suppose $E U_{L}\left(\nu^{L}, \gamma^{L}\right)=\hat{p}\left(R-\nu^{L}-\gamma^{L}\right)+\beta$ where $\frac{\partial \beta}{\partial \mu^{L}} \geq 0$.

Then, following the same logic used in the proof of Proposition $3, L$ 's optimal $\gamma^{L}$ is

$$
\lambda=\frac{\partial \hat{p}}{\partial \gamma^{L}}\left(R-\nu^{L}-\gamma^{L}\right)-\hat{p}+\frac{\partial \beta}{\partial \gamma^{L}}
$$

The most likely case for $L$ to set $\gamma^{L}=0$ is when $W>S-P$, which allows $L$ to assemble a winning coalition without including any members of $P$, while $S$ is sufficiently large to effectively remove the impact of $\gamma^{L}$ on $\hat{\alpha}_{i}^{L}$ for $i \in S / P$ by driving $\bar{p}-\underline{p}$, and thus $\frac{\partial \hat{p}}{\partial \gamma^{L}}$, arbitrarily close to 0 .

If $\frac{\partial \beta}{\partial \mu^{L}}$ is sufficiently large, then since $\frac{\partial \mu^{L}}{\partial \gamma^{L}} \geq 0$, even as the first term in 7 gets arbitrarily close to 0 , the impact of $\frac{\partial \beta}{\partial \gamma^{L}}$ will still outweigh the negative impact of $\hat{p}$. 


\section{References}

Achen, Christopher and Larry Bartles. N.d. "Blind Retrospection: Electoral Responses to Drought, Flu, and Shark Attacks." Draft.

Anderson, Sally and Mark Souva. 2010. "The Accountability Effects of Political Institutions and Capitalism on Interstate Conflict." Journal of Conflict Resolution 54(4):543-565.

Babst, Dean. 1964. "Elective Governments - A Force for Peace." The Wisconsin Sociologist $3(1): 9-14$.

Bennett, D. Scott. 2006. "Towards a Continuous Specification of the Democracy-Autocracy Connection." International Studies Quarterly 50(2):313-338.

Bremer, Stuart. 1992. "Dangerous Dyads: Conditions Affecting the Likelihood of Interstate War, 1816 - 1965." Journal of Conflict Resolution 36(2):309-341.

Bueno de Mesquita, Bruce and Alastair Smith. 2007. "Foreign Aid and Policy Concessions." Journal of Conflict Resolution 51(2):251-284.

Bueno de Mesquita, Bruce and Alastair Smith. 2009. "A Political Economy of Aid." International Organization 63(2):309-340.

Bueno de Mesquita, Bruce, Alastair Smith, Randolph Siverson and James Morrow. 2003. The Logic of Political Survival. Cambridge: The MIT Press.

Bueno de Mesquita, Bruce, James D. Morrow, Randolph M. Siverson and Alastair Smith. 1999. "An Institutional Explanation of the Democratic Peace." American Political Science Review 93(4):791-807.

Chiozza, Giacomo and H.E. Goemans. 2004. "International Conflict and the Tenure of Leaders: Is War Still Ex Post Inefficient?" American Journal of Political Science 48(3):604-619. 
Clarke, Kevin and Randall Stone. 2008. "Democracy and The Logic of Political Survival." American Political Science Review 102(3):387-391.

Collier, Paul and Anke Hoeffler. 2004. "Greed and Grievance in Civil War." Oxford Economic Papers 56(4):563-595.

Danilovic, Vesna and Joe Clare. 2007. "The Kantian Liberal Peace (Revisited)." American Journal of Political Science 51(2):397-414.

Debs, Alexandre and H.E. Goemans. 2010. "Regime Type, the Fate of Leaders, and War." American Political Science Review 104(3):430-445.

Dorussen, Han and Hugh Ward. 2008. "Intergovernmental Organizations and the Kantian Peace: A Network Perspective." Journal of Conflict Resolution 52(2):189-212.

Dorussen, Han and Hugh Ward. 2010. "Trade Networks and the Kantian Peace." 47(1):29$\underline{42 .}$

Edlin, Arron, Andrew Gelman and Noah Kaplan. 2007. "Voting as a Rational Choice: Why and How People Vote to Improve the Well-Being of Others." Rationality and Society 19(3):293-314.

Fearon, James. 1994. "Domestic Political Audiences and the Escalation of International Disputes." American Political Science Review 88(3):577-592.

Fearon, James. 1995. "Rationalist Explanations for War." International Organization 49(3):379-414.

Fearon, James. N.d. "A Simple Political Economy of Relations Between Democracies and Autocracies." Draft.

Fearon, James and David Laitin. 2003. "Ethnicity, Insurgency and Civil War." American Political Science Review 97(1):275-301. 
Feddersen, Timothy, Sean Gailmard and Alvaro Sandroni. 2009. "Moral Bias in Large Elections: Theory and Experimental Evidence." American Political Science Review 103(2):175-192.

Fowler, James. 2006. "Altruism and Turnout." The Journal of Politics 68(3):674-683.

Gartzke, Eric. 2007. "The Capitalist Peace." American Journal of Political Science 51(1):166-191.

Gartzke, Erik, Quan Li and Charles Boehmer. 2001. "Investing in the Peace: Economic Interdependence and International Conflict." International Organization 55(2):391-438.

Gibler, Douglas. 2007. "Bordering on Peace: Democracy, Territorial Issues, and Conflict." International Studies Quarterly 51(3):131-147.

Goemans, Hein. 2000. War and Punishment: The Causes of War Termination and the First World War. Princeton, NJ: Princeton University Press.

Grafstein, Robert. 2009. "The Puzzle of Weak Pocketbook Voting." Journal of Theoretical Politics 21(4):451-482.

Hegre, Håvard. 2001. "Toward a Democratic Civil Peace? Democracy, Political Change, and Civil War, 1816-1992." American Political Science Review 95(1):33-48.

Henderson, Errol. 2002. Democracy and War: The End of an Illusion? Boulder: Lynne Reinner.

Henderson, Errol. 2009. "Disturbing the Peace: African Warfare, Political Inversion and the Universality of the Democratic Peace Thesis." British Journal of Political Science $\underline{39(1): 25-58 .}$

Huth, Paul and Todd Allee. 2002. The Democratic Peace and Territorial Conflict in the Twentieth Century. Cambridge: Cambridge University Press. 
Jackson, Matthew and Massimo Morelli. 2007. "Political Bias and War." American Economic Review 97(4):1353-1373.

Li, Quan and Rafael Reuveny. 2006. "Democracy and Environmental Degradation." International Studies Quarterly 50(4):278-297.

Maoz, Zeev and Bruce Russett. 1993. "Normative and Structural Causes of Democratic Peace, 1946 - 1986." American Political Science Review 87(3):624-638.

McCarty, Nolan and Adam Meirowitz. 2007. Political Game Theory. Cambridge: Cambridge University Press.

McGillivray, Fiona and Alastair Smith. 2006. "Credibility in Compliance and Punishment: Leader Specific Punishments and Credibility." Journal of Politics 68(2):248-258.

McGillivray, Fiona and Alastair Smith. 2008. Punishing the Prince: A Theory of International Relations, Political Institutions and Leader Change. Princeton: Princeton University Press.

Morrow, James, Bruce Bueno de Mesquita, Randolph Siverson and Alastair Smith. 2006. "Selection institutions and war aims." Economics of Governance 7(1):31-52.

Morrow, James, Bruce Bueno de Mesquita, Randolph Siverson and Alastair Smith. 2008. "Retesting Selectorate Theory: Separating the Effects of W from Other Elements of Democracy." American Political Science Review 102(3):393-400.

Mueller, Dennis. 2003. Public Choice III. Cambridge: Cambridge University Press.

Nelson, Joan. 2007. "Elections, democracy, and social services." Studies in Comparative International Development (SCID) 41:79-97.

Oneal, John and Bruce Russett. 1997. "The Classic Liberals Were Right: Democracy, Interdependence and Conflict, 1950 - 1985." International Studies Quarterly 41(2):267-294. 
Oneal, John, Bruce Russett and Michael Berbaum. 2003. "Causes of Peace: Democracy, Interdependence, and International Organizations, 1885-1992." International Studies Quarterly 47(3):371-393.

Patty, John and Roberto Weber. 2006. "Agreeing to Fight: an Explanation of the Democratic Peace." Politics, Philosophy and Economics 5(3):305-320.

Powell, Robert. 1999. In the Shadow of Power. Princeton: Princeton University Press.

Przeworski, Adam, Michael Alvarez, Jose Antonio Cheibub and Fernando Limongi. 2000. Democracy and Development: Political Institutions and Well-Being in the World, 19501990. Cambridge: Cambridge University Press.

Ray, James Lee. 1995. Democracies in International Conflict. Columbia: University of South Carolina Press.

Ray, James Lee. 2003. A Lakatosian View of the Democratic Research Program. In Progress in International Relations Theory: Appraising the Field, ed. Colin Elman and Miriam Fendius Elman. Cambridge: The MIT Press.

Reiter, Dan and Allan C. Stam. 2002. Democracies at War. Princeton, NJ: Princeton University Press.

Riker, William and Peter Ordeshook. 1968. "A Theory of the Calculus of Voting." American Political Science Review 62(1):25-42.

Rosato, Sebastian. 2003. "The Flawed Logic of Democratic Peace Theory." American Political Science Review 97(4):585-602.

Ross, Michael. 2006. "Is Democracy Good for the Poor?" American Journal of Political Science 50(4):860-874.

Russett, Bruce and John R. Oneal. 2001. Triangulating Peace: Democracy, Interdependence, and International Organizations. New York, NY: W.W. Norton \& Company. 
Schultz, Kenneth. 2001. Democracy and Coercive Diplomacy. Cambridge: Cambridge University Press.

Scruggs, Lyle. N.d. "Democracy and Environmental Protection: An Empirical Analysis." Draft Manuscript.

Slantchev, Branislav. 2005. "Military Coercion in Interstate Crises." American Political Science Review 99(4):533-547.

Slantchev, Branislav. 2006. "Politicians, the Media, and Domestic Audience Costs." International Studies Quarterly 50(2):445-477.

Smith, Alastair and Bruce Bueno de Mesquita. N.d. "Pivotal Patronage." Draft manuscript.

Tarar, Ahmer. N.d. "Is the War-as-a-Game-Ending-Costly-Lottery Assumption Distorting?" Draft manuscript.

Tarar, Ahmer and Bahar Leventoğlu. 2008. "Does Private Information Lead to Delay or War in Crisis Bargaining?" International Studies Quarterly 52(3):533-553.

Waltz, Kenneth. 1979. Theory of International Politics. New York: McGraw-Hill.

Ward, Michael, Randolph Siverson and Xun Cao. 2007. "Disputes, Democracies and Dependencies: A Reexamination of the Kantian Peace." American Journal of Political Science 51(3):583-601.

Weeks, Jessica. 2008. "Autocratic Audience Costs: Regime Type and Signaling Resolve." International Organization 62(1):35-64. 